Braz J Med Biol Res, September 2012, Volume 45(9) 856-861

doi: 10.1590/S0100-879X2012007500098

Memory mood congruency phenomenon in bipolar I disorder and major depression disorder patients

V.B. Delgado, F. Kapczinski and M.L.F. Chaves

The Brazilian Journal of Medical and Biological Research is partially financed by

\section{Q Q}

Ministério da Ciência e Tecnologia

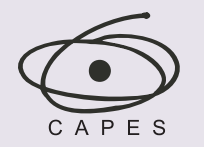

Ministério da Educação
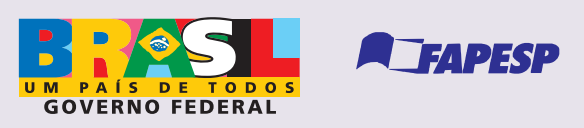

Institutional Sponsors

Ф SHIMADZU UNICAMP

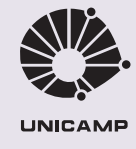

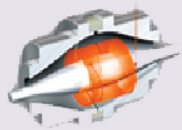

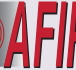

Associaçoo Fundo
delncentio de Incentivo
à Pesquisa

plore High - Performance MS Orbitrap Technology andilicica Thermo
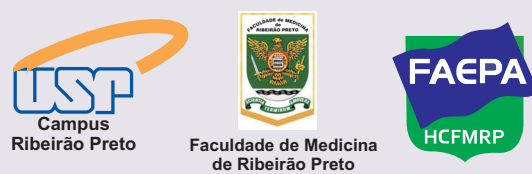


\title{
Memory mood congruency phenomenon in bipolar I disorder and major depression disorder patients
}

\author{
V.B. Delgado ${ }^{1,2}$, F. Kapczinski ${ }^{1,4}$ and M.L.F. Chaves ${ }^{1,3}$ \\ ${ }^{1}$ Programa de Pós-Graduação em Ciências Biológicas: Bioquímica, \\ Universidade Federal do Rio Grande do Sul, Porto Alegre, RS, Brasil \\ 2 Serviço de Enfermagem Psiquiátrica, Hospital de Clínicas de Porto Alegre, Porto Alegre, RS, Brasil \\ ${ }^{3}$ Departamento de Medicina Interna, Faculdade de Medicina, \\ Universidade Federal do Rio Grande do Sul and Serviço de Neurologia, \\ Hospital de Clínicas de Porto Alegre, Porto Alegre, RS, Brasil \\ ${ }^{4}$ Programa de Transtorno Bipolar e Unidade de Psiquiatria Molecular, \\ Hospital de Clínicas de Porto Alegre and Departamento de Psiquiatria, \\ Faculdade de Medicina, Universidade Federal do Rio Grande do Sul, Porto Alegre, RS, Brasil
}

\begin{abstract}
The objective of the present study was to evaluate memory performance in tasks with and without affective content (to confirm the mood congruency phenomenon) in acutely admitted patients with bipolar I disorder (BD) and major depression disorder (MDD) and in healthy participants. Seventy-eight participants (24 BD, 29 MDD, and 25 healthy controls) were evaluated. Three word lists were used as the memory task with affective content (positive, negative and indifferent). Psychiatric symptoms were also evaluated with rating scales (Young Mania Rating Scale for mania and Hamilton Depression Rating Scale for depression). Patients were selected during the first week of hospitalization. BD patients showed higher scores in the word span with positive tone than MDD patients and healthy controls $(P=0.002)$. No other difference was observed for tests with affective tone. MDD patients presented significantly lower scores in the Mini-Mental State Exam, logical memory test, visual recognition span, and digit span, while BD patients presented lower scores in the visual recognition test and digit span. Mood congruency effect was found for word span with positive tone among BD patients but no similar effect was observed among MDD patients for negative items. MDD patients presented more memory impairment than BD patients, but BD patients also showed memory impairment.
\end{abstract}

Key words: Memory; Bipolar disorder; Depression; Affect

\section{Introduction}

Bipolar disorder (BD) is characterized by disturbances in mood ranging from extreme elation (mania) to severe depression often accompanied by psychotic features and cognitive changes (1). There are two types of diagnosis: bipolar I disorder and bipolar II disorder. Bipolar I disorder is characterized by recurrent episodes of mania and depression, while bipolar II disorder is defined as recurrent episodes of depression and hypomania (2). Bipolar I disorder is equally prevalent in men and women, whereas many studies have shown that there are more women than men with bipolar II disorder (3). Prevalence rates have been estimated to be $0.4-1.6 \%$ for bipolar I disorder and $0.5-1.9 \%$ for bipolar II disorder (2). However, when the spectrum of bipolarity is extended to BD in general, the affected population is about $5 \%$ (4). BD is a genetically and neurochemically based complex, recurrent, and potentially progressive neuropsychiatric disorder involving multiple brain systems at the level of neurochemistry, physiology, and structure (5). The precise cause of BD is not known. Historically, dopaminergic models of BD have been dichotomous and global, with mania considered to be a hyperdopaminergic state throughout the brain and depression the reverse of this state (6).

Major depressive disorder (MDD) is a heterogeneous, highly prevalent, and moderately heritable disorder. According to the Diagnostic and Statistical Manual of Mental

Correspondence: M.L.F. Chaves, Rua Ramiro Barcelos, 2350, Sala 2040, 90035-903 Porto Alegre, RS, Brasil.

Fax: +55-51-3388-5085. E-mail: mchaves@hcpa.ufrgs.br

Received March 12, 2012. Accepted June 5, 2012. Available online June 22, 2012. Published August 17, 2012. 
Disorders, 4th edn. (DSM-IV), the diagnosis of MDD requires a minimum of five symptoms (at least one being mood or anhedonia) for a minimum of 2 weeks. The lifetime prevalence of unipolar MDD is at least $10 \%$, with the risk among women being twice the risk for men (7). There are no race differences, and, in general, the occurrence is higher among those without close relationships, divorced or separated (8). Recurrence and early age at onset characterize cases with the greatest familial risk. Most genetic studies have focused on functional polymorphisms (DNA sequence variations that alter the expression and/or functioning of the gene product) in the loci encoding the serotonin transporter (SLC6A4), serotonin 2 A receptor (5HTR2A), tyrosine hydroxylase $(\mathrm{TH}$; the limiting enzyme for dopamine synthesis), tryptophan hydroxylase 1 (TPH1; serotonin synthesis), and catecholo-methyltransferase (COMT; dopamine catabolism) (9). A recent etiological hypothesis is that neurotoxic effects (possibly related to excessive corticotrophin activity and/or to the inflammatory effects of cytokines) on hippocampal cells mediate many depressive symptoms with deficient function of neuroprotective peptides (9). Brain-derived neurotrophic factor (BDNF) is a neuroprotective protein. Initial reports have shown reduced serum BDNF in MDD (10) and association between polymorphisms in BDNF and BD (10). However, subsequent studies have not corroborated such findings uniformly for both disorders (11).

BD patients clearly exhibit extensive neurocognitive dysfunction during acute episodes of mania or depression; however, the demonstration that these deficits endure during remission has raised the possibility that cognitive impairment may represent a trait rather than a state (12). Euthymic $\mathrm{BD}$ patients showed limitations in a number of cognitive domains, especially executive function, declarative memory, and sustained attention (13). MDD is associated with cognitive dysfunction, in particular episodic memory impairment (14). The observed memory deficits may be explained by the association with functional and structural changes in brain structures, including the hippocampus and prefrontal cortex that are critical for episodic memory (15).

Mood is a relatively long-lasting emotional state. Affect is the emotional experience immediately raised by an experience. During a manic episode, the mood is elevated. Patients feel happy, without problems and are not sympathetic to somebody else's feelings, but when they are submitted to frustration they can be short-tempered. Patients show high energy, hyperactivity, distractibility, may be involved in many activities, loss of ideas, pressure to talk, and reduction of sleep. Self-esteem is increased and there are ideas of grandiosity (2). During a depressive episode, a patient presents mood or affect change, can feel depressed, sad and hopeless, report anxiety and agitation, loss of selfesteem or feelings of guilt or depreciation, and reduction of appetite and activities, suicidal ideas or attempts, and sleep disorders (2).

The importance of mood or affect for memory is sig- nificant because performance in memory tests may be influenced by the type of information to be processed or by the affective state of the individual at the time of the test (4). The memory performance of depressed patients in different tasks may be influenced by the affective state, with these patients presenting better acquisition and/or evocation of negative information compared to normal individuals (16). The main evidence of the negative bias of depression was derived from studies with recollection of personal experiences during a depressive episode or studies with the same type of recall during mood induction $(4,17)$.

In state dependency, processing of information is influenced by mood during acquisition, and recall is highest if the mood state is the same. In mood congruency, information is better stored if the affective content corresponds to the subject's affective state (5). In a study that evaluated the acute effect of diazepam on explicit memory with and without affective content in patients with major depression, no anterograde amnesia was observed following diazepam (18). The authors hypothesized that a dysfunction of limbic prefrontal cortical structures that impair the modulation of the amygdala in major depression could explain these results. No equivalent information is available for altered states of mood during mania episodes in BD (state dependency or mood congruency).

The hypothesis raised in the present study is that the mood congruence phenomenon would be present towards elation in BD patients and towards depression in MDD patients. We also intended to demonstrate that acutely manic BD patients would present impaired memory/attention performance compared to healthy participants, but not compared to acutely depressed MDD patients. Therefore, the objective of the present study was to evaluate memory performance in tasks with and without affective content (to verify the mood congruence phenomenon) in acutely admitted patients with type I BD and MDD, and in a group of healthy participants.

\section{Material and Methods}

\section{Subjects and inclusion/exclusion criteria}

A cross-sectional study was carried out to evaluate inpatients with type I BD during a mania episode, and patients with MDD. The sample was composed of 78 participants (24 BD, 29 MDD, and 25 healthy controls). The inclusion of patients followed the DSM-IV criteria for BD and MDD. Exclusion criteria were severe cognitive deficit [Mini-Mental State Examination (MMSE) <10], legal or illegal substance abuse (alcohol and drugs), illiteracy, and age $<20$ and $>60$ years.

Patients were admitted to the Psychiatric Unit of a general university hospital, Hospital de Clínicas de Porto Alegre (Porto Alegre, RS, Brazil) for treatment during an acute exacerbation of illness. The average number of episodes among MD patients was 3.19 , while duration of 
disease was 6.74 years. Among BD patients, the number of episodes was 5.19 and duration of disease was 8.99 years. Use of medication is presented in Table 1. Healthy controls were recruited randomly from the community where the hospital is located.

A battery of cognitive tests was administered to assess attention and memory. Testing began with a visual recognition memory task. Assessment of each participant lasted about 1 $\mathrm{h}$. The same instructions were given to all subjects to prevent subtle differences of interaction with the experimenter.

\section{Cognitive testing and symptom rating scales}

A set of attention/memory tests with affective content (positive, negative, and indifferent) and without affective content was selected to evaluate the attention/memory performance of the participants. The selected tests have been previously adapted and validated for the Brazilian population (19-24).

1) Word span: word lists with emotional content, with 10 items each, presented at a rate of one word/second starting with the positive span and presented consecutively. Higher scores represent better performance. The task evaluates verbal episodic memory $(19,20)$.

2) Wechsler's logical memory test (immediate and delayed recall): a short story with 10 items is presented auditorily to evaluate attention and verbal episodic memory $(21,22)$. Higher scores represent better performance.

3) Digit span: the test starts with two consecutive commands of 3 digits, which are progressively increased up to 2 commands of 10 digits. The test is interrupted when a participant fails to correctly repeat two consecutive commands. Higher scores represent better attention/memory processing. The test evaluates attentional processing, sustained attention and working memory $(21,23)$.

4) Visuospatial recognition span: white round tokens are displayed on a black board, starting from 1 and increasing up to 20 tokens. Each time a token is added to the board, the board is covered. The examinee has to identify the last added token (places are marked and the sequence is fixed). Higher scores mean better performance. This is a task developed to evaluate sustained visual attention and visual memory (24).

Symptoms were rated in each group with the Hamilton Depression Rating Scale (HDRS) (25) and with the Young Mania Rating Scale (YMRS) for mania (26). The 17-item version was used for the HDRS scale and the Portugueseadapted version was used for the YMRS (27). The MMSE was also applied to the 3 groups to exclude cognitive impairment (28).

The study was approved by the Research Ethics Committee of the Hospital de Clínicas de Porto Alegre and all participants gave written informed consent.

\section{Statistical analysis \\ Descriptive statistics (means \pm SD and relative fre-}

quency) were calculated for demographic data, MMSE, and memory tests. Spearman's correlation coefficients were calculated for correlations between the Hamilton and Young scales, number of affective episodes, duration of illness, and memory tests. A univariate general linear model (two-way ANOVA with the Bonferroni post hoc test) was designed for the evaluation of the effects of group (depression, mania, controls), number of affective episodes and memory test scores (ANCOVA with the Bonferroni post hoc test). The statistical analysis was carried out using the Statistical Package for the Social Sciences for Windows (SPSS 14).

\section{Results}

\section{Demographic data}

To evaluate the severity of BD and MDD symptoms we applied the YMRS and the HDRS. The scores on the HDRS were 27.9 for the MDD group, and the scores on the YMRS were 29.3 for the BD group (Table 1).

MDD patients were significantly older and less educated than BD patients (Table 1). Females were more prevalent in all groups studied. The MMSE scores were significantly lower among MDD patients (means \pm SD, $25.56 \pm 2.94$ ) than among BD patients $(27.40 \pm 1.67)$ and healthy controls $(28.23 \pm 1.82)$.

\section{Memory tests with affective content}

In the word span with positive tone, the BD group showed higher scores than the MDD and healthy control groups (Table 2). A significant effect of the number of affective episodes was observed in this test (ANCOVA, negative correlation; $B=-0.13 ; P=0.035)$. The word lists with indifferent and negative tones did not show significant differences among groups.

\section{Memory tests without affective content}

MDD patients presented significantly lower scores in the Digit span than BD and healthy controls (Table 2). A significant effect of number of affective episodes was also observed on Digit span (ANCOVA, negative correlation; B $=-0.21 ; P=0.043$ ). MDD and BD patients presented significantly lower scores than healthy controls in the visuospatial recognition span $(P=0.002$; Table 2$)$. No effect of number of affective episodes was observed.

In the Logical Memory test, the MDD group presented lower scores for immediate recall than healthy controls, but did not differ from the BD group (Table 2). In the delayed recall, both groups of patients (MDD and BD) presented a significantly worse performance than the healthy control group. No effect of number of affective episodes was observed.

\section{Correlations}

Age correlated significantly with word span positive content (rho $=-0.25 ; P=0.030)$, digit span (rho $=-0.28 ; P$ 
Table 1. Demographic and clinical data of the subjects studied.

\begin{tabular}{|c|c|c|c|}
\hline Variables & $\operatorname{MDD}(\mathrm{N}=29)$ & $\mathrm{BD}(\mathrm{N}=24)$ & Healthy controls $(\mathrm{N}=25)$ \\
\hline Age (years) & $45.03 \pm 9.00^{a}$ & $36.83 \pm 12.74^{b}$ & $39.32 \pm 12.22$ \\
\hline Education (years) & $8.14 \pm 2.98^{c}$ & $10.46 \pm 3.60^{d}$ & $9.80 \pm 3.21$ \\
\hline Gender (Female) & $28(96.6 \%)$ & $15(62.5 \%)$ & $19(76.0 \%)$ \\
\hline Duration of illness (months) & $80.91 \pm 118.35$ & $107.91 \pm 80.70$ & - \\
\hline MMSE & $25.56 \pm 2.94^{\mathrm{e}}$ & $27.40 \pm 1.67^{f}$ & $28.23 \pm 1.82^{g}$ \\
\hline HDRS & $27.90 \pm 4.43$ & - & - \\
\hline YMRS & - & $29.33 \pm 3.30$ & - \\
\hline \multicolumn{4}{|l|}{ Medication } \\
\hline SSRI and antipsychotic & $9(31 \%)$ & - & - \\
\hline Tricyclic, benzodiazepine and antipsychotic & $6(21 \%)$ & - & - \\
\hline Lithium carbonate & - & $5(21 \%)$ & - \\
\hline Lithium carbonate, benzodiazepine and SSRI & $4(14 \%)$ & - & - \\
\hline Lithium carbonate and antipsychotic & - & $9(37.5 \%)$ & - \\
\hline $\begin{array}{l}\text { Benzodiazepine, antipsychotic, SSRI and } \\
\text { anticonvulsant }\end{array}$ & $4(14 \%)$ & - & - \\
\hline SSRI & $4(14 \%)$ & - & - \\
\hline MAOI and/or antipsychotic and anticonvulsant & $2(7 \%)$ & - & - \\
\hline Antipsychotic and anticonvulsant & - & $5(21 \%)$ & - \\
\hline
\end{tabular}

Data are reported as means $\pm S D$ or number with percent in parentheses. $a \neq b(P=0.029$, Bonferroni post hoc test); $c \neq d(P=0.031$, Bonferroni post hoc test); $e \neq f ; e \neq g(P<0.002$, Bonferroni post hoc test). MDD = major depression disorder; $\mathrm{BD}=$ bipolar I disorder; MMSE = Mini-Mental State Examination; HDRS = Hamilton Depression Rating Scale; YMRS = Young Mania Rating Scale; SSRI = selective serotonin reuptake inhibitors; $\mathrm{MAOI}=$ monoamine oxidase inhibitors.

$=0.015)$, and visuospatial span (rho $=-0.26 ; P=0.020)$. Education correlated with logical memory immediate recall (rho $=0.26 ; \mathrm{P}=0.024$ ), word span negative content $(r h o=0.38$; $\mathrm{P}=0.001$ ), and digit span (rho = $0.27 ; P=0.016)$. Duration of disease correlated with Word span positive content among BD patients (rho = $-0.48 ; P=0.022$ ).

\section{Discussion}

This study was carried out to analyze performance in memory tasks with and without affective content in acutely manic BD patients, acutely depressed MDD patients, and in a group of healthy controls.

Type I BD patients presented higher scores in the word span with positive content than MDD patients and healthy controls. This finding suggests the hypothesis of the memory mood congruency among BD patients. Mood and affect are important for memory because they may influence performance according to the type of information to be processed or according to the affective state of the studied.
Table 2. Comparison of the scores (means \pm SEM) of memory tests among the groups

\begin{tabular}{lcll}
\hline Tests & MDD $(\mathrm{N}=29)$ & $\mathrm{BD}(\mathrm{N}=24)$ & Healthy controls $(\mathrm{N}=25)$ \\
\hline $\begin{array}{l}\text { Logical memory } \\
\text { Immediate recall }\end{array}$ & $6.0 \pm 2.34^{\mathrm{a}}$ & $6.6 \pm 1.40$ & $7.5 \pm 1.56^{\mathrm{b}}$ \\
$\quad$ Delayed recall & $5.5 \pm 2.49^{\mathrm{c}}$ & $6.0 \pm 1.53^{\mathrm{d}}$ & $7.2 \pm 1.50^{\mathrm{e}}$ \\
Word span & & & \\
$\quad$ Positive tone & $4.4 \pm 1.42^{\mathrm{f}}$ & $5.6 \pm 1.21 \mathrm{~g}$ & $4.9 \pm 1.24^{\mathrm{h}}$ \\
$\quad$ Negative tone & $5.1 \pm 1.72$ & $5.6 \pm 1.43$ & $5.4 \pm 1.08$ \\
$\quad$ Indifferent tone & $4.9 \pm 1.32$ & $5.3 \pm 1.56$ & $5.8 \pm 1.16$ \\
Digit span & $3.7 \pm 2.19^{\mathrm{i}}$ & $5.7 \pm 2.24 \mathrm{j}$ & $5.8 \pm 2.01^{\mathrm{l}}$ \\
Visuospatial recognition span & $7.1 \pm 3.50^{\mathrm{c}}$ & $6.6 \pm 2.42^{\mathrm{d}}$ & $9.8 \pm 3.13^{\mathrm{e}}$ \\
\hline
\end{tabular}

The number of episodes was controlled in the analysis as a covariant. $a \neq b ; c, d \neq e ; g \neq f, h$; $\mathrm{i} \neq \mathrm{j}, \mathrm{I}(\mathrm{P}<0.042, \mathrm{P}<0.017, \mathrm{P}<0.002, \mathrm{P}<0.001$, respectively; ANCOVA with Bonferroni post hoc test). $\mathrm{MDD}=$ major depression disorder; $\mathrm{BD}=$ bipolar I disorder.

individual at the time of the test $(4,17,29)$. Therefore, in mood congruency the information is better stored if the affective content corresponds to the subject's affective state (4), as observed in the present study. No similar information was previously available for altered states of mood and memory processing during manic episodes in $\mathrm{BD}$ (state dependency or mood congruency). The performance in the word span 
with positive content was affected by the number of affective episodes (as demonstrated by ANCOVA) and by the duration of disease (as shown by the Spearman correlation). Thus, we may assume that the task was influenced by the severity of BD (the more severe the disorder the lower the scores in the test). In BD, and depending on the severity of the disease (number of episodes and duration of disease), the positive biased memory processing and the symptoms of the disease (i.e., mania) may be interconnected - one reinforcing the other.

The memory performance of depressed patients has been shown to be influenced by the affective state with better acquisition and/or evocation of negative information $(16,30)$. In our study, no mood congruency effect for negative items was found for MDD patients. No influence of number of episodes or duration of disease was observed in this group. The negative bias of depression was derived from studies with recollection of personal experiences during a depressive episode or studies with the same type of recall during mood induction $(4,16,17,29)$.

MDD patients presented poorer memory performance than healthy participants in the following tests: logical memory immediate and delayed recall, digit span, and visuospatial recognition span. These patients also showed worse performance than BD patients in the digit span and word span with positive tone. In addition to memory tests, MDD patients also presented lower scores in the MMSE, corroborating previous data on cognitive impairment in depressed patients $(14,31)$. Because depression is a frequent and disabling disorder often characterized by a recurrent and chronic course (32), it is well established that depressive disorders are associated with cognitive dysfunction, especially episodic memory impairment $(33,34)$. Among the explanations for these cognitive impairments in depression, the possible association with functional and structural

\section{References}

1. Dias VV, Brissos S, Frey BN, Andreazza AC, Cardoso C, Kapczinski F. Cognitive function and serum levels of brainderived neurotrophic factor in patients with bipolar disorder. Bipolar Disord 2009; 11: 663-671.

2. World Health Organization. International statistical classification of diseases and related health problems ICD-10. Tenth revision, 3rd Vol., 2nd edn., 2004.

3. Martinez-Aran A, Torrent C, Tabares-Seisdedos R, Salamero M, Daban C, Balanza-Martinez V, et al. Neurocognitive impairment in bipolar patients with and without history of psychosis. J Clin Psychiatry 2008; 69: 233-239.

4. Jorm AF, Henderson AS. Memory bias in depression: implications for risk factors studies relying on selfreports of exposure. Int J Methods Psychiat 1992; 2: 31-38.

5. Merikangas KR, Akiskal HS, Angst J, Greenberg PE, Hirschfeld RM, Petukhova M, et al. Lifetime and 12-month prevalence of bipolar spectrum disorder in the National Comorbidity Survey replication. Arch Gen Psychiatry 2007; changes in brain structures (i.e., hippocampus and prefrontal cortex - critical for episodic memory) is central $(15,35)$.

We also found other memory impairments among BD patients. The performance in the logical memory delayed recall and in the visuospatial recognition span was lower compared to that of the healthy participants. There is now much evidence that patients with BD show cognitive impairment during the acute phases of the illness, which persists during inter-episode periods, even when mood is euthymic $(36,37)$. Attentional processing, executive function, and verbal memory are the cognitive functions usually impaired in BD (38). Impairment in some domains (visual and working memory, and risk-taking behavior) did not show remission during periods of euthymia, while it did show remission in others (selective attention, attentional shifting, verbal memory, verbal planning, processing speed, and the elements of executive function such as inhibitory control, response inhibition, or strategic thought) (39).

The large proportion of women among depressed patients is one of the limitations of the study; however, this was evaluated in the statistical analysis. On the other hand, this investigation presents several strengths such as the evaluation of acutely hospitalized patients, the determination of disease severity with worldwide rating scales, and the use of a healthy group from the same community as that of the patients.

We detected the mood congruency effect for the word span with positive content among BD patients but no similar effect among MDD patients for negative items. MDD patients presented more memory impairments than BD patients, while BD patients also showed memory impairments compared to the healthy participants. Indeed, bipolar patients did not resemble depressed patients in the performance of different memory tasks, but cognitive difficulties in bipolar patients may help explain impairment of daily functioning.
64: 543-552.

6. Cousins DA, Butts $\mathrm{K}$, Young AH. The role of dopamine in bipolar disorder. Bipolar Disord 2009; 11: 787-806.

7. Weissman MM, Bland RC, Canino GJ, Faravelli C, Greenwald $\mathrm{S}$, Hwu HG, et al. Cross-national epidemiology of major depression and bipolar disorder. JAMA 1996; 276: 293-299.

8. Kaplan HI, Sadoch BJ. Comprehensive textbook of psychiatry modern synopsis of comprehensive psychiatry. 7nd edn. Baltimore: Williams \& Wilkins; 1990.

9. Levinson DF. The genetics of depression: a review. Biol Psychiatry 2006; 60: 84-92.

10. Karege F, Perret G, Bondolfi G, Schwald M, Bertschy G, Aubry JM. Decreased serum brain-derived neurotrophic factor levels in major depressed patients. Psychiatry Res 2002; 109: 143-148.

11. Hashimoto K, Shimizu E, lyo M. Critical role of brain-derived neurotrophic factor in mood disorders. Brain Res Brain Res 
Rev 2004; 45: 104-114.

12. Glahn DC, Therman S, Manninen M, Huttunen M, Kaprio $\mathrm{J}$, Lonnqvist J, et al. Spatial working memory as an endophenotype for schizophrenia. Biol Psychiatry 2003; 53: 624-626.

13. Kurtz MM, Gerraty RT. A meta-analytic investigation of neurocognitive deficits in bipolar illness: profile and effects of clinical state. Neuropsychology 2009; 23: 551-562.

14. Airaksinen E, Larsson M, Lundberg I, Forsell Y. Cognitive functions in depressive disorders: evidence from a population-based study. Psychol Med 2004; 34: 83-91.

15. Campbell S, Marriott M, Nahmias C, MacQueen GM. Lower hippocampal volume in patients suffering from depression: a meta-analysis. Am J Psychiatry 2004; 161: 598-607.

16. Teasdale JD, Russell ML. Differential effects of induced mood on the recall of positive, negative and neutral words. Br J Clin Psychol 1983; 22 (Part 3): 163-171.

17. Blaney PH. Affect and memory: a review. Psychol Bull 1986; 99: 229-246.

18. Delgado VB, Izquierdo I, Chaves ML. Differential effects of acute diazepam on emotional and neutral memory tasks in acutely hospitalized depressed patients. Neuropsychiatr Dis Treat 2005; 1: 269-275.

19. Ceitlin LHS, Santos BJ, Parizotto I, Parizotto I, Zanatta MS, Chaves MLF. Elaboration of word lists in Portuguese with emotional content and their influence on memory function in normal subjects. Int J Meth Psychiatric Res 1995; 4: 121129.

20. Bertolucci PH, Okamoto IH, Brucki SM, Siviero MO, Toniolo NJ, Ramos LR. Applicability of the CERAD neuropsychological battery to Brazilian elderly. Arq Neuropsiquiatr 2001; 59: $532-536$

21. Wechsler D. Wechsler memory scale-revised. San Antonio: Psychol Cooperation; 1987.

22. Chaves ML, Iha D, Maia AL, Motta E, Lehmen R, Oliveira LM. Diagnosing dementia and normal aging: clinical relevance of brain ratios and cognitive performance in a Brazilian sample. Braz J Med Biol Res 1999; 32: 1133-1143.

23. Chaves ML, Izquierdo I. Differential diagnosis between dementia and depression: a study of efficiency increment. Acta Neurol Scand 1992; 85: 378-382.

24. Rebok G, Brandt J, Folstein M. Longitudinal cognitive decline in patients with Alzheimer's disease. J Geriatr Psychiatry Neurol 1990; 3: 91-97.

25. Hamilton M. A rating scale for depression. J Neurol Neurosurg Psychiatry 1960; 23: 56-62.

26. Young RC, Biggs JT, Ziegler VE, Meyer DA. A rating scale for mania: reliability, validity and sensitivity. $B r J$ Psychiatry 1978; 133: 429-435.

27. Vilela JAA. Estudo da confiabilidade e validade de uma versão modificada da Young Mania Rating Scale. [Masters Dissertation]: Faculdade de Medicina de Ribeirão Preto; 2000.

28. Folstein MF, Folstein SE, McHugh PR. "Mini-mental state". A practical method for grading the cognitive state of patients for the clinician. J Psychiatr Res 1975; 12: 189-198.

29. Blackburn IM, Roxborough HM, Muir WJ, Glabus M, Blackwood DH. Perceptual and physiological dysfunction in depression. Psychol Med 1990; 20: 95-103.

30. Teasdale JD, Fogarty SJ. Differential effects of induced mood on retrieval of pleasant and unpleasant events from episodic memory. J Abnorm Psychol 1979; 88: 248-257.

31. Burt DB, Zembar MJ, Niederehe G. Depression and memory impairment: a meta-analysis of the association, its pattern, and specificity. Psychol Bull 1995; 117: 285-305.

32. Ormel J, Oldehinkel AJ, Nolen WA, Vollebergh W. Psychosocial disability before, during, and after a major depressive episode: a 3-wave population-based study of state, scar, and trait effects. Arch Gen Psychiatry 2004; 61: 387-392.

33. Austin MP, Mitchell P, Goodwin GM. Cognitive deficits in depression: possible implications for functional neuropathology. Br J Psychiatry 2001; 178: 200-206.

34. Airaksinen E, Wahlin A, Larsson M, Forsell Y. Cognitive and social functioning in recovery from depression: results from a population-based three-year follow-up. J Affect Disord 2006; 96: 107-110.

35. Drevets WC. Neuroimaging studies of mood disorders. Biol Psychiatry 2004; 8: 829.

36. Robinson LJ, Thompson JM, Gallagher P, Goswami U, Young $\mathrm{AH}$, Ferrier IN, et al. A meta-analysis of cognitive deficits in euthymic patients with bipolar disorder. J Affect Disord 2006; 93: 105-115.

37. Martinez-Aran A, Vieta E, Colom F, Torrent C, SanchezMoreno J, Reinares M, et al. Cognitive impairment in euthymic bipolar patients: implications for clinical and functional outcome. Bipolar Disord 2004; 6: 224-232.

38. Ferrier IN, Chowdhury R, Thompson JM, Watson S, Young $\mathrm{AH}$. Neurocognitive function in unaffected first-degree relatives of patients with bipolar disorder: a preliminary report. Bipolar Disord 2004; 6: 319-322.

39. Dixon T, Kravariti E, Frith C, Murray RM, McGuire PK. Effect of symptoms on executive function in bipolar illness. Psychol Med 2004; 34: 811-821. 